QUARTERLY OF APPLIED MATHEMATICS

VOLUME LXVII, NUMBER 4

DECEMBER 2009, PAGES 687-705

S 0033-569X(09)01133-X

Article electronically published on May 27, 2009

\title{
ASYMPTOTIC STABILITY FOR THREE-DIMENSIONAL LINEAR DIFFERENTIAL SYSTEMS WITH TIME-VARYING COEFFICIENTS
}

\author{
BY \\ JITSURO SUGIE (Department of Mathematics and Computer Science, Shimane University, Matsue \\ 690-8504, Japan) \\ AND \\ YUICHI OGAMI (Department of Mathematics and Computer Science, Shimane University, Matsue \\ 690-8504, Japan)
}

\begin{abstract}
This paper is concerned with the asymptotic stability of the zero solution of three-dimensional linear differential systems with variable coefficients. The coefficients are not assumed to be positive. A concept innovated by László Hatvani plays a vital role in our results. Sufficient conditions are also given for the zero solution to be uniformly stable. Some suitable examples are included to illustrate our results. Finally, certain changes of variable are used to broaden the application of our results.
\end{abstract}

1. Introduction. In this paper, we present sufficient conditions for the zero solution of time-varying three-dimensional linear differential systems to be asymptotically stable.

As for time-invariant linear differential systems, the study of stability can date back to the nineteenth century. It goes without saying that the steam engine invented by J. Watt played a leading role in the Industrial Revolution. The steam engine continued being improved by the development of the Industrial Revolution. The velocity of a machine is measured and regulated by a centrifugal speed regulator called a governor. A governor plays the role which keeps the operation speed of a steam engine constant. In the process of the design change of the steam engine, most governors stopped acting well and the operation speed started changing continuously. Destabilization of the operation speed is called the hunting phenomenon, and it was a great blow to the industrial world. Many scientists and engineers did their best to escape from this social predicament. J. C. Maxwell, who is famous for the Maxwell equations, ascertained this cause first. He described the structure of governors in 1868 by a third-order linear differential equation

Received May 8, 2008.

2000 Mathematics Subject Classification. Primary 34D05, 34D20, 34D23; Secondary 37B25, 37B55.

Key words and phrases. Uniform stability, asymptotic stability, linear differential systems, weakly integrally positive.

The first author was supported in part by Grant-in-Aid for Scientific Research, No. 19540182.

E-mail address: jsugie@riko.shimane-u.ac.jp 
(see [8]) and succeeded in the analysis of the hunting phenomenon of governors. Maxwell's result was generalized by E. J. Routh who was a classmate of Maxwell and a stability criterion for the $n$-th order linear differential equation was given (see [12]). About 20 years later, without knowing Routh's method, A. Hurwitz gave another stability criterion expressed simply by determinants (see [6]). It was shown later that Routh's method and Hurwitz's method are equivalent although the forms are different from each other. At present, their methods are called the Routh-Hurwitz criterion. This stability criterion is still used as an effective basic theorem in control engineering.

Applying the Routh-Hurwitz stability criterion to the time-invariant linear differential equation

$$
x^{\prime \prime \prime}+a x^{\prime \prime}+b x^{\prime}+c x=0,
$$

we can easily see that $a>0$ and $a b>c>0$ are necessary and sufficient conditions for the equilibrium $\left(x, x^{\prime}, x^{\prime \prime}\right)=(0,0,0)$ of (1.1) to be asymptotically stable (for the definition, see Section 2). Judging from this fact, it might be inferred that the equilibrium of the equation

$$
x^{\prime \prime \prime}+a(t) x^{\prime \prime}+b(t) x^{\prime}+c(t) x=0
$$

is asymptotically stable if

$$
a(t)>0 \quad \text { and } \quad a(t) b(t)>c(t)>0 \text { for } t \geq 0 .
$$

However, this assertion is not true. In fact, consider the equation

$$
x^{\prime \prime \prime}+\frac{3}{1+t} x^{\prime \prime}+(2+t) x^{\prime}+\frac{1}{1+t} x=0 .
$$

Then, assumption (1.3) is satisfied with $a(t)=3 /(1+t), b(t)=2+t$ and $c(t)=1 /(1+t)$, but equation (1.4) has a solution $x(t)=\mu(2+t) /(1+t)$, where $\mu$ is an arbitrary number. Since the solution does not tend to zero unless $\mu=0$, the equilibrium of (1.4) is not asymptotically stable.

There are a number of transformations into a system equivalent to (1.1). For example, let $y=x^{\prime}$ and $z=x^{\prime \prime}$ be new variables. Then equation (1.1) becomes the system

$$
x^{\prime}=y, \quad y^{\prime}=z, \quad z^{\prime}=-c x-b y-a z,
$$

which is called the companion form. Let $y=x^{\prime}$ and $z=x^{\prime \prime}+c x / a$. Then equation (1.1) becomes the system

$$
x^{\prime}=y, \quad y^{\prime}=-\frac{c}{a} z, \quad z^{\prime}=-\left(b-\frac{c}{a}\right) y-a z .
$$

System (1.6) is usually called Schwarz's form, which is convenient for verifying the RouthHurwitz stability criterion. The equilibrium of (1.1) corresponds to the zero solution $(x(t), y(t), z(t))=(0,0,0)$ of (1.5) or (1.6).

Systems (1.5) and (1.6) naturally have constant coefficients. As shown by the fact that assumption (1.3) is insufficient for the equilibrium of (1.2) to be asymptotically stable, if we change from constant coefficients to time-varying coefficients, then it is very hard to analyze the asymptotic stability of the zero solution of a system of the companion form or Schwarz's form. 
To avoid this difficulty, we would like to propose a new form. We will direct our attention to an equivalent system of the form

$$
x^{\prime}=\sqrt{\frac{c}{a}} y, \quad y^{\prime}=-\sqrt{\frac{c}{a}} x, \quad z^{\prime}=-\left(b-\frac{c}{a}\right) y-a z .
$$

In equation (1.2), we assume that $a(t)$ and $c(t)$ are continuously differentiable, and $b(t)$ is continuous for $t \geq 0$. Let

$$
e^{2}(t)=\exp (-2 A(t) / 3)\left\{d+\frac{2}{3} \int_{0}^{t} c(s) \exp (2 A(s) / 3) d s\right\}
$$

where $A(t)=\int_{0}^{t} a(s) d s$ and $d$ is an arbitrary positive number. Then, letting

$$
y=\frac{1}{e(t)} x^{\prime} \quad \text { and } \quad z=\frac{1}{e(t)} x^{\prime \prime}-\frac{e^{\prime}(t)}{e^{2}(t)} x^{\prime}+e(t) x,
$$

we have the following system equivalent to (1.2):

$$
x^{\prime}=e(t) y, \quad y^{\prime}=-e(t) x+f(t) z, \quad z^{\prime}=-g(t) y-h(t) z,
$$

where $f(t)=1$,

$$
g(t)=b(t)-e^{2}(t)-\frac{1}{3} a^{\prime}(t)-\frac{2}{9} a^{2}(t)+\frac{a(t) c(t)}{3 e^{2}(t)}+\frac{c^{\prime}(t)}{3 e^{2}(t)}-\frac{c^{2}(t)}{9 e^{4}(t)}
$$

and

$$
h(t)=\frac{1}{3} a(t)+\frac{2 c(t)}{3 e^{2}(t)}
$$

for $t \geq 0$ (for details, see Section 4). System (1.8) has the same form as (1.7). In the case that $a(t)=a>0, b(t)=b>0$ and $c(t)=c>0$ for $t \geq 0$, system (1.8) coincides with (1.7) (we may choose $c / a>0$ as $d$ ).

As for system (1.8), what kind of condition on time-varying coefficients $e(t), f(t), g(t)$ and $h(t)$ will guarantee that the zero solution is asymptotically stable? To assure the asymptotic stability of the equilibrium of (1.2), in addition to assumption (1.3), what kind of condition will be required? The purpose of this paper is to answer these questions.

In Section 2, we state our main result on the asymptotic stability of the zero solution of (1.8). To this end, we make several assumptions on the coefficients $e(t), f(t), g(t)$ and $h(t)$. Also, we give sufficient conditions for the zero solution of (1.8) to be uniformly stable. In Section 3, we prove our main result. For illustration of our main theorem, we take some concrete examples and those figures of a positive orbit in Section 4. Finally, we present some corollaries.

2. Assumptions. Consider a system of differential equations of the form

$$
x^{\prime}=e(t) y, \quad y^{\prime}=-e(t) x+f(t) z, \quad z^{\prime}=-g(t) y-h(t) z,
$$

where the coefficients $e(t), f(t), g(t)$ and $h(t)$ are continuous and $g(t) / f(t)$ is differentiable for $t \geq 0$.

Let $\mathbf{x}(t)=(x(t), y(t), z(t))$ and $\mathbf{x}_{0}=\left(x_{0}, y_{0}, z_{0}\right) \in \mathbb{R}^{3}$, and let $\|\cdot\|$ be the Euclidean norm. We denote the solution of (E) through $\left(t_{0}, \mathbf{x}_{0}\right)$ by $\mathbf{x}\left(t ; t_{0}, \mathbf{x}_{0}\right)$. It is clear that system $(E)$ has the zero solution $\mathbf{x}(t) \equiv \mathbf{0}$. 
The zero solution is said to be stable, if for any $\varepsilon>0$ and any $t_{0} \geq 0$, there exists a $\delta\left(\varepsilon, t_{0}\right)>0$ such that $\left\|\mathbf{x}_{0}\right\|<\delta$ implies $\left\|\mathbf{x}\left(t ; t_{0}, \mathbf{x}_{0}\right)\right\|<\varepsilon$ for all $t \geq t_{0}$. The zero solution is said to be uniformly stable if it is stable and $\delta$ can be chosen to be independent of $t_{0}$. The zero solution is said to be attractive if for any $t_{0} \geq 0$, there exists a $\delta_{0}\left(t_{0}\right)>0$ such that $\left\|\mathbf{x}_{0}\right\|<\delta_{0}$ implies $\left\|\mathbf{x}\left(t ; t_{0}, \mathbf{x}_{0}\right)\right\| \rightarrow 0$ as $t \rightarrow \infty$. For homogeneous linear differential systems such as $(E)$, if the zero solution is attractive, then it is stable. The zero solution of $(E)$ is said to be asymptotically stable if it is attractive. The asymptotic stability and the attractivity are completely different concepts in general systems.

We also assume that $f(t)$ and $g(t)$ are bounded for $t \geq 0$ and that

(i) $f(t) g(t)>0$ for $t \geq 0$ and $\liminf _{t \rightarrow \infty} f(t) g(t)>0$.

Then there exist positive numbers $k$ and $K$ such that

$$
k \leq \frac{f(x)}{g(x)} \leq K \quad \text { for } t \geq 0 .
$$

In fact, since $f(t)$ and $g(t)$ are bounded for $t \geq 0$, there exist constants $c_{1}>0$ and $c_{2}>0$ such that

$$
|f(t)| \leq c_{1} \quad \text { and } \quad|g(t)| \leq c_{2}
$$

for $t \geq 0$. Because of assumption (i), there exists a $c_{3}>0$ with

$$
f(t) g(t) \geq c_{3} \text { for } t \geq 0 \text {. }
$$

Hence, it is clear that

$$
\frac{f(t)}{g(t)}=\frac{f(t) g(t)}{g^{2}(t)} \geq \frac{c_{3}}{c_{2}^{2}} \quad \text { for } t \geq 0 .
$$

We can find a number $c_{4}>0$ satisfying

$$
|g(t)| \geq c_{4} \quad \text { for } t \geq 0
$$

If the assertion is false, then there exists a sequence $\left\{t_{n}\right\}$ tending to $\infty$ such that

$$
\left|g\left(t_{n}\right)\right| \rightarrow 0 \quad \text { as } n \rightarrow \infty
$$

It then follows from (2.3) that

$$
\left|f\left(t_{n}\right)\right| \rightarrow \infty \text { as } n \rightarrow \infty \text {. }
$$

This contradicts (2.2). We therefore conclude that

$$
\frac{f(t)}{g(t)}=\frac{|f(t)|}{|g(t)|} \leq \frac{c_{1}}{c_{4}} \quad \text { for } t \geq 0 .
$$

Let $k=c_{3} / c_{2}^{2}$ and $K=c_{1} / c_{4}$. Then we obtain the inequality (2.1).

Since $g(t) / f(t)$ is differentiable for $t \geq 0$, we may define

$$
\psi(t)=2 h(t)+\frac{f(t)}{g(t)}\left(\frac{g(t)}{f(t)}\right)^{\prime} .
$$

For the sake of simplicity, we denote

$$
\psi_{+}(t)=\max \{0, \psi(t)\} \quad \text { and } \quad \psi_{-}(t)=\max \{0,-\psi(t)\} .
$$

Then we have the following result. 
LEMMA 2.1. In addition to assumption (i), if

(ii) $\int_{0}^{\infty} \psi_{-}(s) d s<\infty$,

then the zero solution of $(E)$ is uniformly stable.

Proof. Let $\mathbf{x}=(x, y, z)$ and define

$$
V(t, \mathbf{x})=\frac{1}{2}\left(x^{2}+y^{2}+\frac{f(t)}{g(t)} z^{2}\right) \quad \text { and } \quad W(t, \mathbf{x})=V(t, \mathbf{x}) \exp (-\Psi(t))
$$

on $[0, \infty) \times \mathbb{R}^{3}$, where

$$
\Psi(t)=\int_{0}^{t} \psi_{-}(s) d s .
$$

Because of assumption (ii), there exists an $L>0$ such that $\Psi(t)<L$ for $t \geq 0$. Hence, together with the inequality (2.1), we have

$$
\begin{aligned}
\frac{1}{2}\left(x^{2}+y^{2}+k z^{2}\right) e^{-L} & \leq V(t, \mathbf{x}) e^{-L} \leq W(t, \mathbf{x}) \\
& \leq V(t, \mathbf{x}) \leq \frac{1}{2}\left(x^{2}+y^{2}+K z^{2}\right)
\end{aligned}
$$

Differentiate $V(t, \mathbf{x})$ along any solution of (E) obtaining

$$
\dot{V}_{\mathbb{E \mathbb { E }}}(t, \mathbf{x})=-\frac{1}{2} \psi(t) \frac{f(t)}{g(t)} z^{2} \leq \frac{1}{2} \psi_{-}(t) \frac{f(t)}{g(t)} z^{2} \leq \psi_{-}(t) V(t, \mathbf{x}) .
$$

Hence, we get

$$
\dot{W}_{\underline{E(E)}}(t, \mathbf{x})=\left\{\dot{V}_{\underline{E(E)}}(t, \mathbf{x})-\psi_{-}(t) V(t, \mathbf{x})\right\} \exp (-\Psi(t)) \leq 0
$$

for $t \geq 0$ and $\mathbf{x} \in \mathbb{R}^{3}$. Thus, $W(t, \mathbf{x})$ is positive definite and decrescent, and $\dot{W}_{\mathbb{E}(}(t, \mathbf{x})$ is nonpositive. We therefore conclude that the zero solution of $(E)$ is uniformly stable by using a Liapunov-type theorem due to Persidskir [10] (as to the direct method of Liapunov, for example, see the books [1, 2, 1, 9, 11, 14]).

For the sake of brevity, let $\mathbf{x}(t)=\mathbf{x}\left(t ; t_{0}, \mathbf{x}_{0}\right)$ and $v(t)=V(t, \mathbf{x}(t))$. Then, as shown in the proof of Lemma 2.1, we have

$$
v^{\prime}(t) \leq \psi_{-}(t) v(t) \text { for } t \geq t_{0} .
$$

The inequality (2.4) guarantees the convergence of $v(t)$.

Lemma 2.2. Under the assumption (ii), if (2.4) holds, then $v^{\prime}(t)$ is absolutely integrable, and therefore, $v(t)$ has a nonnegative limiting value.

Proof. By (2.4), we have

$$
v(t) \leq v\left(t_{0}\right) \exp \left(\Psi(t)-\Psi\left(t_{0}\right)\right) \leq v\left(t_{0}\right) e^{L}
$$

for $t \geq t_{0}$, where $L$ is the number given in the proof of Lemma 2.1. Hence, using (2.4) again, we obtain

$$
v^{\prime}(t) \leq v\left(t_{0}\right) e^{L} \psi_{-}(t) \text { for } t \geq t_{0}
$$

Since the right-hand side of this inequality is nonnegative for $t \geq t_{0}$, we get

$$
v_{+}^{\prime}(t) \leq v\left(t_{0}\right) e^{L} \psi_{-}(t)
$$


Integrate both sides from $t_{0}$ to $\infty$ to obtain

$$
\int_{t_{0}}^{\infty} v_{+}^{\prime}(s) d s \leq v\left(t_{0}\right) L e^{L}<\infty .
$$

On the other hand, since $v(t) \geq 0$ for $t \geq t_{0}$, we have

$$
\int_{t_{0}}^{\infty} v_{-}^{\prime}(s) d s \leq v\left(t_{0}\right)+\int_{t_{0}}^{\infty} v_{+}^{\prime}(s) d s<\infty .
$$

We therefore conclude that

$$
\int_{t_{0}}^{\infty}\left|v^{\prime}(s)\right| d s=\int_{t_{0}}^{\infty}\left(v_{+}^{\prime}(s)+v_{-}^{\prime}(s)\right) d s<\infty
$$

as required. This completes the proof of Lemma 2.2.

We have already assumed the continuity of $e(t)$ in the opening paragraph of this section. Let us make an additional assumption on $e(t)$. To this end, we define the union of intervals as follows: for any triplex $(\alpha, \beta, \gamma)$ with $0<\alpha \leq \beta$ and $\gamma>0$, let

$$
I_{t}(\alpha, \beta, \gamma) \stackrel{\text { def }}{=} \bigcup\{[s, s+\alpha] \subset[t, t+\beta]:|e(\tau)| \geq \gamma \text { for } \tau \in[s, s+\alpha]\} .
$$

To take some examples, if $e(t)=1$ for $t \geq 0$, then $I_{t}(\alpha, \beta, \gamma) \equiv[t, t+\beta]$ for any $(\alpha, \beta, \gamma)$ with $0<\alpha \leq \beta$ and $0<\gamma \leq 1$; if $e(t)=\sin t$ for $t \geq 0$, then

$$
I_{t}(2 \pi / 3,2 \pi, 1 / 2)= \begin{cases}{\left[\frac{1}{6} \pi, \frac{5}{6} \pi\right] \cup\left[\frac{7}{6} \pi, \frac{11}{6} \pi\right] \quad} & \text { if } 0 \leq t \leq \frac{1}{6} \pi, \\ {\left[\left(n+\frac{1}{6}\right) \pi,\left(n+\frac{5}{6}\right) \pi\right] \cup\left[\left(n+\frac{7}{6}\right) \pi,\left(n+\frac{11}{6}\right) \pi\right]} & \text { if }\left(n-\frac{1}{6}\right) \pi \leq t \leq\left(n+\frac{1}{6}\right) \pi, \\ {\left[\left(n+\frac{1}{6}\right) \pi,\left(n+\frac{5}{6}\right) \pi\right]} & \text { if }\left(n-\frac{5}{6}\right) \pi<t<\left(n-\frac{1}{6}\right) \pi .\end{cases}
$$

Similarly, if $e(t)$ satisfies that $\liminf _{t \rightarrow \infty}|e(t)|>0$ or if $e(t)$ is a nontrivial periodic function, then $I_{t}(\alpha, \beta, \gamma)$ is not empty for all $t \geq 0$. Needless to say, there are cases where $I_{t}(\alpha, \beta, \gamma)$ is an empty set for any $(\alpha, \beta, \gamma)$. For example, consider the case in which $e(t)=\sin \left(t^{2}\right)$ for $t \geq 0$. Since $e(t)$ is over-oscillation, we cannot choose a suitable constant $\alpha>0$. Hence, $I_{t}(\alpha, \beta, \gamma)$ is empty for $t$ sufficiently large. To show another case that $I_{t}(\alpha, \beta, \gamma)$ is empty, we define a sequence $\left\{d_{n}\right\}$ by $d_{n}=n(n-1) / 2$ for $n \in \mathbb{N}$ and consider the $C^{1}$-function

$$
e(t)=\left\{\begin{array}{cl}
\sin ^{2} t & \text { for } t \in\left[d_{n} \pi,\left(d_{n}+1\right) \pi\right], \\
0 & \text { for } t \in\left[\left(d_{n}+1\right) \pi, d_{n+1} \pi\right] .
\end{array}\right.
$$

In this case, the part where $e(t)>0$ is the sum of the open intervals $\left(d_{n} \pi,\left(d_{n}+1\right) \pi\right)$. The distance between each interval and the next expands as $n$ increases. Hence, for any $\alpha>0$ and $\gamma>0$, we cannot find $\beta$ with $\alpha \leq \beta$ such that $I_{t}(\alpha, \beta, \gamma)$ is nonempty. 
We here assume that

(iii) there exists a triplex $(\alpha, \beta, \gamma)$ with $0<\alpha \leq \beta$ and $\gamma>0$ such that $I_{t}(\alpha, \beta, \gamma)$ is nonempty for $t$ sufficiently large.

Assumption (iii) is satisfied in the case in which $\liminf _{t \rightarrow \infty}|e(t)|>0$ or $e(t)$ is a nontrivial periodic function, including an almost periodic function, but it is not satisfied in the case in which $e(t)=\sin \left(t^{2}\right)$ or $e(t)$ is given by (2.5).

REMARK 2.1. If $e(t)$ satisfies assumption (iii) for a triplex $\left(\alpha_{0}, \beta_{0}, \gamma_{0}\right)$, then it also satisfies assumption (iii) for $(\alpha, \beta, \gamma)$ with $0<\alpha<\alpha_{0}, \beta>\beta_{0}$ and $0<\gamma<\gamma_{0}$.

To state our main result, we have to introduce a concept that plays an important role in this paper. A nonnegative function $\phi$ is said to be integrally positive if

$$
\int_{J} \phi(s) d s=\infty
$$

for every set $J=\bigcup_{n=1}^{\infty}\left[\tau_{n}, \sigma_{n}\right]$ such that $\tau_{n}<\sigma_{n}<\tau_{n+1}$ and $\sigma_{n}-\tau_{n} \geq \lambda>0$. If, in addition, the set $J$ satisfies $\tau_{n+1} \leq \sigma_{n}+\Lambda$ for some $\Lambda>0$, then we say that the function $\phi$ is weakly integrally positive. We can cite $1 /(1+t)$ or $\sin ^{2} t /(1+t)$ as a function that is weakly integrally positive, but not integrally positive (refer to [3, 4, 5, 13]). If $\phi$ is (weakly) integrally positive, then it naturally satisfies

$$
\lim _{t \rightarrow \infty} \int^{t} \phi(s) d s=\infty .
$$

REMARK 2.2. Suppose that $\phi$ is weakly integrally positive. If

$$
\sum_{n=1}^{\infty} \int_{\tau_{n}}^{\sigma_{n}} \phi(s) d s<\infty
$$

for a number $\Lambda>0$ and two sequences $\left\{\tau_{n}\right\}$ and $\left\{\sigma_{n}\right\}$ with $\tau_{n}<\sigma_{n}<\tau_{n+1} \leq \sigma_{n}+\Lambda$, then $\liminf _{n \rightarrow \infty}\left(\sigma_{n}-\tau_{n}\right)=0$.

We are now in a position to present the main result. We will give its proof in the next section.

Theorem 2.3. Let $e(t), f(t), g(t)$ and $h(t)$ be bounded for $t \geq 0$. Suppose that assumptions (i)-(iii) are satisfied. If

(iv) $\psi_{+}(t)$ is weakly integrally positive, then the zero solution of $(E \mathbb{E})$ is asymptotically stable.

3. Proof of the main result. Before proving Theorem 2.3, it is helpful to transform system $(E)$ into an equivalent system. In cylindrical coordinates $(x, y, z) \rightarrow(r, \theta, z)$ by $x=r \cos \theta$ and $y=r \sin \theta$, system (E) takes the form

$$
r^{\prime}=f(t) z \sin \theta, \quad \theta^{\prime}=\frac{f(t) z}{r} \cos \theta-e(t), \quad z^{\prime}=-g(t) r \sin \theta-h(t) z .
$$

Let $(r(t), \theta(t), z(t))$ be the solution of $(\widetilde{\mathbb{E}})$ corresponding to $\mathbf{x}(t)=\mathbf{x}\left(t ; t_{0}, \mathbf{x}_{0}\right)$.

Proof of Theorem 2.3. By means of Lemma 2.1, the zero solution of (E) is uniformly stable, that is, given any $\varepsilon>0$, there exists a $\delta(\varepsilon)>0$ such that $t_{0} \geq 0$ and $\left\|\mathbf{x}_{0}\right\|<\delta$ imply

$$
\|\mathbf{x}(t)\|<\varepsilon \quad \text { for } t \geq t_{0} .
$$


In order to prove that the zero solution of $(E)$ is asymptotically stable, it is enough to show that it is attractive, namely, $\mathbf{x}(t)$ converges to $\mathbf{0}$ as $t$ increases.

For convenience of notation, set

$$
u(t)=\frac{f(t)}{2 g(t)} z^{2}(t) .
$$

Then, by (2.1) we have

$$
v(t)=V(t, \mathbf{x}(t))=\frac{1}{2}\left(x^{2}(t)+y^{2}(t)\right)+u(t) \geq \frac{1}{2}\left(x^{2}(t)+y^{2}(t)+k z^{2}(t)\right)
$$

for $t \geq t_{0}$. Recall that $v(t)$ satisfies

$$
v^{\prime}(t)=-\psi(t) u(t) \leq \psi_{-}(t) v(t)
$$

for $t \geq t_{0}$ (see the calculation of $\dot{V}_{|E|}(t, \mathbf{x})$ in the proof of Lemma 2.1). Hence, from Lemma 2.2 we see that $v(t)$ has a limiting value $v_{0} \geq 0$. If $v_{0}=0$, then by (3.2), the solution $\mathbf{x}(t)$ tends to $\mathbf{0}$ as $t \rightarrow \infty$. This completes the proof. Hereafter, we consider only the case in which $v_{0}>0$. We will demonstrate that this case does not occur.

From (2.1) and (3.1), we see that $u(t)$ is bounded. Hence, $u(t)$ has the inferior limit and the superior limit. First, we will show that the inferior limit of $u(t)$ is zero, and we will then show that the superior limit of $u(t)$ is also zero.

Suppose that $\liminf _{t \rightarrow \infty} u(t)>0$. Then there exist an $\varepsilon_{1}>0$ and a $T_{1} \geq t_{0}$ such that $u(t)>\varepsilon_{1}$ for $t \geq T_{1}$. From (3.3) and Lemma 2.2 it follows that

$$
\infty>\int_{t_{0}}^{\infty}\left|v^{\prime}(s)\right| d s=\int_{t_{0}}^{\infty}|\psi(s)| u(s) d s \geq \int_{t_{0}}^{\infty} \psi_{+}(s) u(s) d s>\varepsilon_{1} \int_{T_{1}}^{\infty} \psi_{+}(s) d s .
$$

This contradicts assumption (iii). Thus, we see that $\liminf _{t \rightarrow \infty} u(t)=0$.

By way of a contradiction, we suppose that $\nu \stackrel{\text { def }}{=} \limsup _{t \rightarrow \infty} u(t)>0$. Since $e(t), f(t)$ and $h(t)$ are bounded for $t \geq 0$, there exist numbers $\bar{e}>0, \bar{f}>0$ and $\bar{h}>0$ such that

$$
|e(t)| \leq \bar{e}, \quad|f(t)| \leq \bar{f} \quad \text { and } \quad|h(t)| \leq \bar{h}
$$

for $t \geq 0$. As mentioned in Section 2, we can choose a $\underline{g}>0$ such that

$$
|g(t)| \geq \underline{g} \text { for } t \geq 0 .
$$

Since $v(t)$ tends to a positive value $v_{0}$ as $t \rightarrow \infty$, there exists a $T_{2} \geq t_{0}$ such that

$$
0<\frac{v_{0}}{2}<v(t)<\frac{3 v_{0}}{2} \text { for } t \geq T_{2} .
$$

Let $\varepsilon_{2}>0$ be so small that $\varepsilon_{2}<\nu / 2$ and

$$
\frac{\sqrt{2 \varepsilon_{2} / k}}{\sqrt{v_{0}-2 \varepsilon_{2}}}<\min \left\{\frac{\gamma}{6 \bar{f}}, \frac{\alpha \gamma \underline{g} \sin (\alpha \gamma / 4)}{2(\gamma+6 \bar{e})+\alpha \gamma \bar{h}}\right\},
$$

where $\alpha$ and $\gamma$ are the numbers given in assumption (iii). Since $\liminf _{t \rightarrow \infty} u(t)=$ $0<\nu=\limsup _{t \rightarrow \infty} u(t)$, we can find two divergent sequences $\left\{\tau_{n}\right\}$ and $\left\{\sigma_{n}\right\}$ with $T_{2}<\tau_{n}<\sigma_{n}<\tau_{n+1}$ such that

$$
\begin{aligned}
u(t) \geq \varepsilon_{2} & \text { for } \tau_{n}<t<\sigma_{n}, \\
0 \leq u(t) \leq \varepsilon_{2} & \text { for } \sigma_{n}<t<\tau_{n+1}
\end{aligned}
$$


and $u\left(\tau_{n}\right)=u\left(\sigma_{n}\right)=\varepsilon_{2}$. Since $u(t) \geq k z^{2}(t) / 2$ for $t \geq t_{0}$, we have

$$
|z(t)| \leq \sqrt{\frac{2}{k} u(t)}<\sqrt{\frac{2 \varepsilon_{2}}{k}} \text { for } \sigma_{n} \leq t \leq \tau_{n+1} .
$$

Using (3.1), (3.2), (3.6) and (3.8), we also obtain

$$
\sqrt{v_{0}-2 \varepsilon_{2}}<\sqrt{2(v(t)-u(t))}=\sqrt{x^{2}(t)+y^{2}(t)}<\varepsilon
$$

for $\sigma_{n} \leq t \leq \tau_{n+1}$, namely,

$$
\sqrt{v_{0}-2 \varepsilon_{2}}<r(t)<\varepsilon \quad \text { for } \sigma_{n} \leq t \leq \tau_{n+1} .
$$

From (3.9) and (3.10), we see that the solution $(r(t), \theta(t), z(t))$ of $(\underline{\mathbb{E}}$ stays in the thin disc

$$
D=\left\{(r, \theta, z): \sqrt{v_{0}-2 \varepsilon_{2}}<r<\varepsilon,-\pi<\theta \leq \pi \text { and }|z| \leq \sqrt{2 \varepsilon_{2} / k}\right\}
$$

for $\sigma_{n} \leq t \leq \tau_{n+1}$.

We will show that $\tau_{n+1}-\sigma_{n} \leq \beta$ for $n \in \mathbb{N}$ sufficiently large, where $\beta$ is the number given in assumption (iii). If the assertion is not true, then there exists a sufficiently large $n_{0} \in \mathbb{N}$ with $\tau_{n_{0}+1}-\sigma_{n_{0}}>\beta$. Because of assumption (iii), there exist two numbers $\omega_{1}$ and $\omega_{2}$ with $\sigma_{n_{0}} \leq \omega_{1}<\omega_{2} \leq \sigma_{n_{0}}+\beta$ and $\omega_{2}-\omega_{1}=\alpha$ such that

$$
|e(t)| \geq \gamma>0 \text { for } \omega_{1} \leq t \leq \omega_{2} .
$$

Taking Remark 2.1 into account, we may regard the product $\alpha \gamma$ as being small, say, $0<\alpha \gamma<2 \pi$. Since $e(t)$ is continuous for $t \geq 0$, there are two cases to consider: (a) $e(t) \geq \gamma$ for $t \in\left[\omega_{1}, \omega_{2}\right]$ and (b) $e(t) \leq-\gamma$ for $t \in\left[\omega_{1}, \omega_{2}\right]$. We consider only the former because the latter can be carried out in the same manner. By (3.4), (3.9) and (3.10), we have

$$
\begin{aligned}
-\frac{\bar{f} \sqrt{2 \varepsilon_{2} / k}}{\sqrt{v_{0}-2 \varepsilon_{2}}}-\bar{e} & <-\frac{|f(t)||z(t)|}{r(t)}-|e(t)| \\
& \leq \theta^{\prime}(t) \leq \frac{|f(t)||z(t)|}{r(t)}-e(t)<\frac{\bar{f} \sqrt{2 \varepsilon_{2} / k}}{\sqrt{v_{0}-2 \varepsilon_{2}}}-\gamma
\end{aligned}
$$

for $\omega_{1} \leq t \leq \omega_{2}$. From (3.7), we see that

$$
-\frac{1}{6} \gamma-\bar{e}<\theta^{\prime}(t)<-\frac{5}{6} \gamma \text { for } \omega_{1} \leq t \leq \omega_{2},
$$

and therefore, we obtain

$$
\frac{5}{6} \alpha \gamma<\theta\left(\omega_{1}\right)-\theta\left(\omega_{2}\right)<\left(\frac{1}{6} \gamma+\bar{e}\right) \alpha .
$$

We now define a pair of regions $\Omega_{+}$and $\Omega_{-}$by

and

$$
\begin{aligned}
& \Omega_{+}=\left\{(r, \theta): \sqrt{v_{0}-2 \varepsilon_{2}}<r<\varepsilon \text { and } \alpha \gamma / 4<\theta<\pi-\alpha \gamma / 4\right\} \\
& \Omega_{-}=\left\{(r, \theta): \sqrt{v_{0}-2 \varepsilon_{2}}<r<\varepsilon \text { and }-\pi+\alpha \gamma / 4<\theta<-\alpha \gamma / 4\right\} .
\end{aligned}
$$

The regions $\Omega_{+}$and $\Omega_{-}$are nonempty because $0<\alpha \gamma<2 \pi$. Since the union of $\Omega_{+}$and $\Omega_{-}$is symmetric with respect to the horizontal line $\theta=0$ and the vertical line $r=0$, we 
may regard $\theta\left(\omega_{1}\right)$ as being in the first quadrant. Consider the movement of $(r(t), \theta(t))$. As already shown, $(r(t), \theta(t))$ stays in the annulus

$$
A=\left\{(r, \theta): \sqrt{v_{0}-2 \varepsilon_{2}}<r<\varepsilon \text { and }-\pi<\theta \leq \pi\right\}
$$

for $\omega_{1} \leq t \leq \omega_{2}$ and it moves clockwise. If $\theta\left(\omega_{1}\right) \in \Omega_{+}$, then there are three possibilities:

- $(r(t), \theta(t))$ remains in $\Omega_{+}$for $\omega_{1} \leq t \leq \omega_{2}$;

- $(r(t), \theta(t))$ leaves $\Omega_{+}$and enters into the region $A \backslash\left(\Omega_{+} \cup \Omega_{-}\right)$; in other words, there exists an $\omega_{3}$ with $\omega_{1}<\omega_{3} \leq \omega_{2}$ such that $(r(t), \theta(t)) \in \Omega_{+}$for $\omega_{1} \leq t<\omega_{3}$ and $(r(t), \theta(t)) \in A \backslash\left(\Omega_{+} \cup \Omega_{-}\right)$for $\omega_{3} \leq t<\omega_{2}$;

- $(r(t), \theta(t))$ passes through the region $A \backslash\left(\Omega_{+} \cup \Omega_{-}\right)$and reaches $\Omega_{-}$; in other words, there exist two numbers $\omega_{3}$ and $\omega_{4}$ with $\omega_{1}<\omega_{3}<\omega_{4}<\omega_{2}$ such that $(r(t), \theta(t)) \in \Omega_{+}$for $\omega_{1} \leq t<\omega_{3},(r(t), \theta(t)) \in A \backslash\left(\Omega_{+} \cup \Omega_{-}\right)$for $\omega_{3} \leq t \leq \omega_{4}$ and $(r(t), \theta(t)) \in \Omega_{-}$for $\omega_{4}<t \leq \omega_{2}$.

If $\theta\left(\omega_{1}\right) \notin \Omega_{+}$, then $(r(t), \theta(t))$ passes through the region $A \backslash\left(\Omega_{+} \cup \Omega_{-}\right)$and enters into $\Omega_{-}$. In this case, it follows from (3.11) that $(r(t), \theta(t))$ does not remain in the region $A \backslash\left(\Omega_{+} \cup \Omega_{-}\right)$for $\omega_{1} \leq t \leq \omega_{2}$. Thus, we can choose an $\omega_{4}$ with $\omega_{1}<\omega_{4}<\omega_{2}$ such that $(r(t), \theta(t)) \in A \backslash\left(\Omega_{+} \cup \Omega_{-}\right)$for $\omega_{1} \leq t \leq \omega_{4}$ and $(r(t), \theta(t)) \in \Omega_{-}$for $\omega_{4}<t \leq \omega_{2}$. To sum up, in all cases, we can find two numbers $\omega_{3}$ and $\omega_{4}$ with $\omega_{1} \leq \omega_{3} \leq \omega_{4} \leq \omega_{2}$ such that $\theta\left(\omega_{3}\right)-\theta\left(\omega_{4}\right) \leq \alpha \gamma / 2$ and

$$
(r(t), \theta(t)) \in \begin{cases}\Omega_{+} & \text {for } \omega_{1}<t<\omega_{3}, \\ \Omega_{-} & \text {for } \omega_{4}<t<\omega_{2}\end{cases}
$$

(in the first case, we should consider that $\omega_{3}=\omega_{4}=\omega_{2}$; in the second case, we should consider that $\omega_{4}=\omega_{2}$; in the fourth case, we should consider that $\left.\omega_{3}=\omega_{1}\right)$. Of course, if $\omega_{1}=\omega_{3}$ (resp., $\left.\omega_{4}=\omega_{2}\right)$, then we regard the interval $\left(\omega_{1}, \omega_{3}\right)$ (resp., $\left(\omega_{4}, \omega_{2}\right)$ ) as an empty set. Using (3.11) again, we have

$$
\begin{aligned}
\frac{5}{6} \alpha \gamma & <\theta\left(\omega_{1}\right)-\theta\left(\omega_{2}\right) \\
& =\left(\theta\left(\omega_{1}\right)-\theta\left(\omega_{3}\right)\right)+\left(\theta\left(\omega_{3}\right)-\theta\left(\omega_{4}\right)\right)+\left(\theta\left(\omega_{4}\right)-\theta\left(\omega_{2}\right)\right) \\
& \leq\left(\theta\left(\omega_{1}\right)-\theta\left(\omega_{3}\right)\right)+\frac{1}{2} \alpha \gamma+\left(\theta\left(\omega_{4}\right)-\theta\left(\omega_{2}\right)\right) .
\end{aligned}
$$

Hence, we conclude that

$$
\max \left\{\theta\left(\omega_{1}\right)-\theta\left(\omega_{3}\right), \theta\left(\omega_{4}\right)-\theta\left(\omega_{2}\right)\right\}>\frac{1}{6} \alpha \gamma .
$$

We may assume without loss of generality that $\theta\left(\omega_{1}\right)-\theta\left(\omega_{3}\right) \geq \theta\left(\omega_{4}\right)-\theta\left(\omega_{2}\right)$. Then it is clear that $\alpha \gamma / 4 \leq \theta\left(\omega_{3}\right) \leq \theta(t) \leq \theta\left(\omega_{1}\right) \leq \pi / 2$ for $\omega_{3} \leq t \leq \omega_{1}$. Hence, it follows that

$$
|\sin \theta(t)|=\sin |\theta(t)| \geq \sin \frac{1}{4} \alpha \gamma \text { for } \omega_{1} \leq t \leq \omega_{3} .
$$

We next estimate the difference between $\omega_{1}$ and $\omega_{3}$. Since $\theta^{\prime}(t)>-\gamma / 6-\bar{e}$, we have

$$
\theta\left(\omega_{1}\right)-\theta\left(\omega_{3}\right)<\left(\frac{1}{6} \gamma+\bar{e}\right)\left(\omega_{3}-\omega_{1}\right) .
$$


Hence, we obtain

$$
\omega_{3}-\omega_{1}>\frac{\theta\left(\omega_{1}\right)-\theta\left(\omega_{3}\right)}{\gamma / 6+\bar{e}}>\frac{\alpha \gamma}{\gamma+6 \bar{e}}
$$

Using (3.4), (3.5), (3.7), (3.9), (3.10), (3.12) and the third equation of (苂), we get

$$
\begin{aligned}
\left|z^{\prime}(t)\right| & \geq|g(t)||r(t)||\sin \theta(t)|-|h(t)||z(t)| \\
& >\underline{g} \sqrt{v_{0}-2 \varepsilon_{2}} \sin \frac{1}{4} \alpha \gamma-\bar{h} \sqrt{\frac{2 \varepsilon_{2}}{k}}>0
\end{aligned}
$$

for $\omega_{1} \leq t \leq \omega_{3}$. Since $z^{\prime}(t)$ is continuous for $t \geq t_{0}$, we see that

$$
\left|\int_{\omega_{1}}^{\omega_{3}} z^{\prime}(s) d s\right|=\int_{\omega_{1}}^{\omega_{3}}\left|z^{\prime}(s)\right| d s .
$$

Hence, by (3.7), (3.9) and (3.13), we have

$$
\begin{aligned}
2 \sqrt{\frac{2 \varepsilon_{2}}{k}} & \geq\left|z\left(\omega_{1}\right)\right|+\left|z\left(\omega_{3}\right)\right| \geq \int_{\omega_{1}}^{\omega_{3}}\left|z^{\prime}(s)\right| d s \\
& >\left(\underline{g} \sqrt{v_{0}-2 \varepsilon_{2}} \sin \frac{1}{4} \alpha \gamma-\bar{h} \sqrt{\frac{2 \varepsilon_{2}}{k}}\right)\left(\omega_{3}-\omega_{1}\right) \\
& >\frac{2(\gamma+6 \bar{e})}{\alpha \gamma} \sqrt{\frac{2 \varepsilon_{2}}{k}}\left(\omega_{3}-\omega_{1}\right)>2 \sqrt{\frac{2 \varepsilon_{2}}{k}},
\end{aligned}
$$

which is a contradiction. Thus, we have verified that $\tau_{n+1}-\sigma_{n} \leq b$ for $n \in \mathbb{N}$ sufficiently large.

Let $J=\bigcup_{n=1}^{\infty}\left[\tau_{n}, \sigma_{n}\right]$. From Lemma 2.2 and (3.8), we see that

$$
\infty>\int_{t_{0}}^{\infty}\left|v^{\prime}(s)\right| d s \geq \int_{t_{0}}^{\infty} \psi_{+}(s) u(s) d s>\varepsilon_{2} \int_{J} \psi_{+}(s) d s .
$$

Hence, taking into account Remark 2.2 with $\Lambda=\beta$, we have

$$
\liminf _{t \rightarrow \infty}\left(\sigma_{n}-\tau_{n}\right)=\infty
$$

Since $\liminf \operatorname{int}_{t \rightarrow \infty} u(t)=0$ and $\lim \sup _{t \rightarrow \infty} u(t)=\nu>0$, we can choose two sequences $\left\{t_{n}\right\}$ and $\left\{s_{n}\right\}$ with $T_{2}<t_{n}<s_{n}<t_{n+1}$ such that $u\left(t_{n}\right)=\nu / 2, u\left(s_{n}\right)=3 \nu / 4$ and

$$
\frac{\nu}{2}<u(t)<\frac{3 \nu}{4} \text { for } t_{n}<t<s_{n}
$$

Since $\varepsilon_{2}<\nu / 2$, we may consider that $\left[t_{n}, s_{n}\right] \subset\left[\tau_{n}, \sigma_{n}\right]$ for $n \in \mathbb{N}$ (if necessary, we can change $\left\{\tau_{n}\right\}$ and $\left\{\sigma_{n}\right\}$ into suitable subsequences of $\left\{\tau_{n}\right\}$ and $\left\{\sigma_{n}\right\}$ ). Hence, by (3.14), we have

$$
\liminf _{n \rightarrow \infty}\left(s_{n}-t_{n}\right)=0 .
$$

From (3.1), (3.2) and (3.4), we see that

$$
u^{\prime}(t)=v^{\prime}(t)-x(t) x^{\prime}(t)-y(t) y^{\prime}(t) \leq\left|v^{\prime}(t)\right|+|f(t)||y(t)||z(t)| \leq\left|v^{\prime}(t)\right|+\bar{f} \varepsilon^{2}
$$

for $t \geq t_{0}$. Integrating this inequality from $t_{n}$ to $s_{n}$, we get

$$
\frac{\nu}{4}=\left|u\left(s_{n}\right)\right|-\left|u\left(t_{n}\right)\right| \leq \int_{t_{n}}^{s_{n}}\left|v^{\prime}(s)\right| d s+\bar{f} \varepsilon^{2}\left(s_{n}-t_{n}\right)
$$


for each $n \in \mathbb{N}$. By (3.15), the right-hand side of the above inequality tends to 0 as $n \rightarrow \infty$. This is a contradiction. We therefore conclude that $\limsup _{t \rightarrow \infty} u(t)=0$.

As proven above, $u(t)$ tends to zero as $t \rightarrow \infty$. Hence, there exists a $T_{3} \geq t_{0}$ such that

$$
0 \leq u(t)<\varepsilon_{2} \text { for } t \geq T_{3} .
$$

Using this estimation instead of (3.8) and repeating the same process once more, we see that the solution $(r(t), \theta(t), z(t))$ of $(\underline{\mathbb{E}})$ stays in the disc $D$ for $t \geq T_{3}$, that is, $(r(t), \theta(t))$ remains in the annulus $A$ for $t \geq T_{3}$. For this reason, we can choose two numbers $\omega_{5}$ and $\omega_{6}$ with $T_{3}<\omega_{5}<\omega_{6}$ such that $(r(t), \theta(t)) \in \Omega_{+}$for $\omega_{5}<t<\omega_{6}$. We can estimate that

$$
\begin{gathered}
\omega_{6}-\omega_{5}>\frac{\theta\left(\omega_{5}\right)-\theta\left(\omega_{6}\right)}{\gamma / 6+\bar{e}}>\frac{\alpha \gamma}{\gamma+6 \bar{e}}, \\
\left|z^{\prime}(t)\right|>\underline{g} \sqrt{v_{0}-2 \varepsilon_{2}} \sin \frac{1}{4} \alpha \gamma-\bar{h} \sqrt{\frac{2 \varepsilon_{2}}{k}}>0
\end{gathered}
$$

for $\omega_{5}<t<\omega_{6}$. Using these estimations, we have

$$
2 \sqrt{\frac{2 \varepsilon_{2}}{k}} \geq \int_{\omega_{5}}^{\omega_{6}}\left|z^{\prime}(s)\right| d s>\left(\underline{g} \sqrt{v_{0}-2 \varepsilon_{2}} \sin \frac{1}{4} \alpha \gamma-\bar{h} \sqrt{\frac{2 \varepsilon_{2}}{k}}\right)\left(\omega_{6}-\omega_{5}\right)>2 \sqrt{\frac{2 \varepsilon_{2}}{k}},
$$

which is a contradiction. Thus, the case of $v_{0}>0$ does not happen. We therefore conclude that the zero solution of $(E)$ is asymptotically stable.

The proof of Theorem 2.1 is thus complete.

4. Discussion and simulation. As a special case of $(E)$, we consider the linear differential system with constant coefficients,

$$
x=e y, \quad y^{\prime}=-e x+f z, \quad z^{\prime}=-g y-h z .
$$

System (4.1) is equivalent to the third-order linear differential equation

$$
x^{\prime \prime \prime}+h x^{\prime \prime}+\left(e^{2}+f g\right) x^{\prime}+e^{2} h x=0 .
$$

By using the Routh-Hurwitz criterion, it can be shown that the zero solution of (4.1) is asymptotically stable if and only if the determinants $\Delta_{1}=h$,

$$
\Delta_{2}=\operatorname{det}\left(\begin{array}{cc}
h & e^{2} h \\
1 & e^{2}+f g
\end{array}\right)=f g h \quad \text { and } \quad \Delta_{3}=\operatorname{det}\left(\begin{array}{ccc}
h & e^{2} h & 0 \\
1 & e^{2}+f g & 0 \\
0 & h & e^{2} h
\end{array}\right)=e^{2} f g h^{2}
$$

are positive, namely, $e \neq 0, f g>0$ and $h>0$. We will confirm that assumptions (i)-(iv) in Theorem 2.1 are satisfied. If $f$ and $g$ have like signs, then assumption (i) is clearly satisfied. Since $\psi(t)=2 h$ for system (4.1), $\psi_{-}(t)=0$ and $\psi_{+}(t)=2 h$. Hence, assumptions (ii) and (iv) are satisfied for $h>0$. If $e \neq 0$, then $I_{t}(\alpha, \beta, \gamma)$ exists for any $0<\alpha<\beta$ and $0<\gamma \leq|e|$. Hence, assumption (iii) is also satisfied. Thus, by Theorem 2.1, we conclude that if $e \neq 0, f g>0$ and $h>0$, then the zero solution of (4.1) is asymptotically stable. For the reason above, it is safe to say that our main theorem is considerably sharp.

To illustrate our main theorem, we give a simple example. 

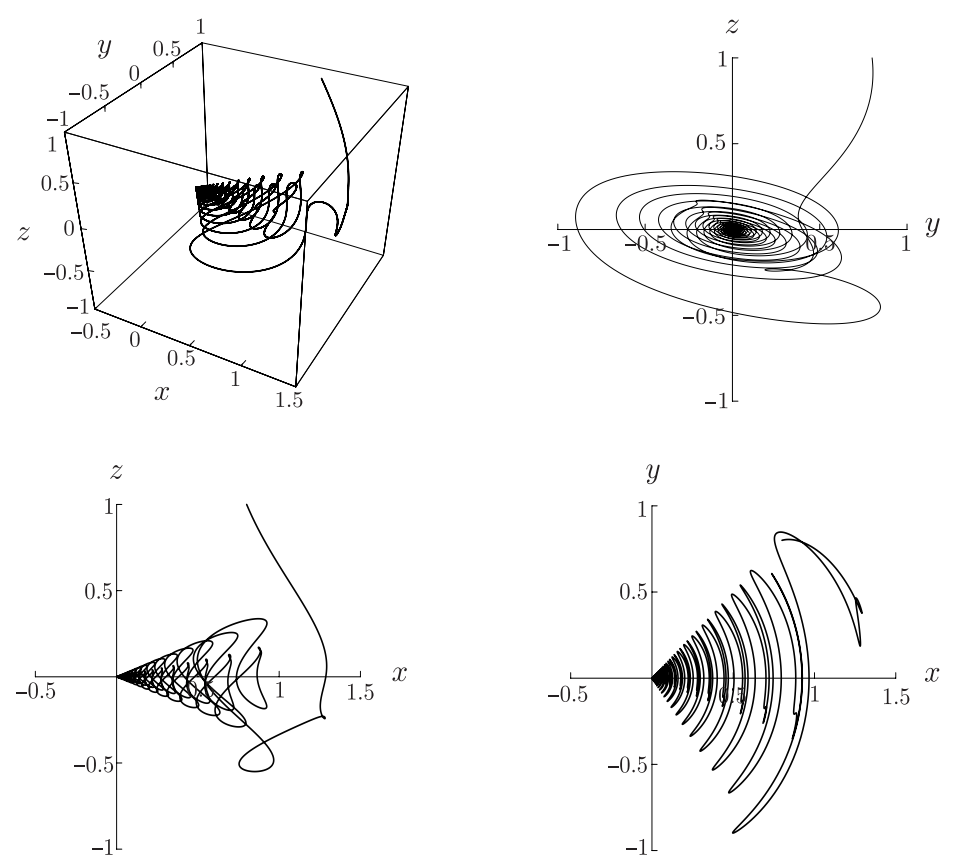

FIG. 1. A positive orbit of E with (4.2)

Example 4.1. Consider system (E) with

$$
e(t)=\sin 2 t+\cos 3 t \quad \text { and } \quad f(t)=g(t)=h(t)=1 .
$$

Then the zero solution of $(\underline{E})$ is asymptotically stable.

Assumptions (i)-(iii) are clearly satisfied. Since $e(t)$ is periodic, assumption (iv) also holds. Hence, by Theorem 2.1, we see that the zero solution of $(E)$ is asymptotically stable. In Figure 1, we draw a positive orbit of (E) with (4.1) and its projections onto the $(x, y)$-plane, the $(y, z)$-plane and the $(x, z)$-plane. The initial time $t_{0}=0$ and the starting point $\left(x_{0}, y_{0}, z_{0}\right)=(0.8,0.8,1)$. The positive orbit approaches the origin $(0,0,0)$ by a regular but somewhat complicated motion.

The form of system $(E)$ is by no means restrictive. We will show that the main result can be applied to the third-order linear differential equation

$$
x^{\prime \prime \prime}+a(t) x^{\prime \prime}+b(t) x^{\prime}+c(t) x=0
$$

where $a(t)$ and $c(t)$ are continuously differentiable, and $b(t)$ is continuous for $t \geq 0$. If $a(t), b(t)$ and $c(t)$ are the constants $a, b$ and $c$, respectively, then $a>0$ and $a b>c>0$ are necessary and sufficient constants for the equilibrium $\left(x, x^{\prime}, x^{\prime \prime}\right)=(0,0,0)$ to be asymptotically stable. Hence, it may safely be assumed that

$$
a(t)>0 \text { and } a(t) b(t)>c(t)>0 \text { for } t \geq 0 .
$$


Denote $A(t)=\int_{0}^{t} a(s) d s$ and let

$$
e^{2}(t)=\exp (-2 A(t) / 3)\left\{d+\frac{2}{3} \int_{0}^{t} c(s) \exp (2 A(s) / 3) d s\right\},
$$

where $d$ is an arbitrary positive number. Then $e^{2}(0)=d$ and $e^{2}(t)$ is a positive $C^{2}$ function for $t \geq 0$. Hence, $e(t) \neq 0$ for $t \geq 0$. As new variables, let

$$
y=\frac{1}{e(t)} x^{\prime} \quad \text { and } \quad z=\frac{1}{e(t)} x^{\prime \prime}-\frac{e^{\prime}(t)}{e^{2}(t)} x^{\prime}+e(t) x .
$$

Then it is clear that $x^{\prime}=e(t) y$ and $y^{\prime}=-e(t) x+f(t) z$, where $f(t) \equiv 1$. From (4.4), we see that

$$
\frac{e^{\prime}(t)}{e(t)}=-\frac{1}{3} a(t)+\frac{1}{3} \frac{c(t)}{e^{2}(t)}
$$

Hence, we have

$$
z=\frac{1}{e(t)} x^{\prime \prime}+\frac{1}{3}\left(\frac{a(t)}{e(t)}-\frac{c(t)}{e^{3}(t)}\right) x^{\prime}+e(t) x .
$$

By (4.3) and a straightforward computation, we obtain

$$
\begin{aligned}
z^{\prime}= & \frac{1}{e(t)} x^{\prime \prime \prime}-\frac{e^{\prime}(t)}{e^{2}(t)} x^{\prime \prime}+\frac{1}{3}\left(\frac{a(t)}{e(t)}-\frac{c(t)}{e^{3}(t)}\right) x^{\prime \prime} \\
& +\frac{1}{3}\left(\frac{a^{\prime}(t)}{e(t)}-\frac{a(t) e^{\prime}(t)}{e^{2}(t)}-\frac{c^{\prime}(t)}{e^{3}(t)}+\frac{3 c(t) e^{\prime}(t)}{e^{4}(t)}\right) x^{\prime}+e(t) x^{\prime}+e^{\prime}(t) x \\
= & -\frac{a(t)}{e(t)} x^{\prime \prime}-\frac{b(t)}{e(t)} x^{\prime}-\frac{c(t)}{e(t)} x+\frac{2}{3}\left(\frac{a(t)}{e(t)}-\frac{c(t)}{e^{3}(t)}\right) x^{\prime \prime} \\
& +\frac{1}{9}\left(\frac{3 a^{\prime}(t)}{e(t)}+\frac{a^{2}(t)}{e(t)}-\frac{4 a(t) c(t)}{e^{3}(t)}-\frac{3 c^{\prime}(t)}{e^{3}(t)}+\frac{3 c^{2}(t)}{e^{5}(t)}\right) x^{\prime}+e(t) x^{\prime}+e^{\prime}(t) x \\
= & -\frac{1}{3}\left(\frac{a(t)}{e(t)}+\frac{2 c(t)}{e^{3}(t)}\right) x^{\prime \prime}-\frac{b(t)}{e(t)} x^{\prime} \\
& +\frac{1}{9}\left(\frac{3 a^{\prime}(t)}{e(t)}+\frac{a^{2}(t)}{e(t)}-\frac{4 a(t) c(t)}{e^{3}(t)}-\frac{3 c^{\prime}(t)}{e^{3}(t)}+\frac{3 c^{2}(t)}{e^{5}(t)}\right) x^{\prime}+e(t) x^{\prime} \\
& -\frac{1}{3}\left(a(t) e(t)+\frac{2 c(t)}{e(t)}\right) x \\
= & -\left(b(t)-e^{2}(t)-\frac{1}{3} a^{\prime}(t)-\frac{2}{9} a^{2}(t)+\frac{a(t) c(t)}{3 e^{2}(t)}+\frac{c^{\prime}(t)}{3 e^{2}(t)}-\frac{c^{2}(t)}{9 e^{4}(t)}\right) \frac{1}{e(t)} x^{\prime} \\
& -\frac{1}{3}\left(\frac{a(t)}{e(t)}+\frac{2 c(t)}{e^{3}(t)}\right) x^{\prime \prime}-\frac{1}{9}\left(\frac{a^{2}(t)}{e(t)}+\frac{a(t) c(t)}{e^{3}(t)}-\frac{2 c^{2}(t)}{e^{5}(t)}\right) x^{\prime} \\
& -\frac{1}{3}\left(a(t) e(t)+\frac{2 c(t)}{e(t)}\right) x
\end{aligned}
$$




$$
\begin{aligned}
= & -\left(b(t)-e^{2}(t)-\frac{1}{3} a^{\prime}(t)-\frac{2}{9} a^{2}(t)+\frac{a(t) c(t)}{3 e^{2}(t)}+\frac{c^{\prime}(t)}{3 e^{2}(t)}-\frac{c^{2}(t)}{9 e^{4}(t)}\right) \frac{1}{e(t)} x^{\prime} \\
& -\frac{1}{3}\left(a(t)+\frac{2 c(t)}{e^{2}(t)}\right)\left(\frac{1}{e(t)} x^{\prime \prime}+\frac{1}{3}\left(\frac{a(t)}{e(t)}-\frac{c(t)}{e^{3}(t)}\right) x^{\prime}+e(t) x\right) \\
= & -\left(b(t)-e^{2}(t)-\frac{1}{3} a^{\prime}(t)-\frac{2}{9} a^{2}(t)+\frac{a(t) c(t)}{3 e^{2}(t)}+\frac{c^{\prime}(t)}{3 e^{2}(t)}-\frac{c^{2}(t)}{9 e^{4}(t)}\right) y \\
& -\frac{1}{3}\left(a(t)+\frac{2 c(t)}{e^{2}(t)}\right) z \\
= & -g(t) y-h(t) z,
\end{aligned}
$$

where

$$
g(t)=b(t)-e^{2}(t)-\frac{1}{3} a^{\prime}(t)-\frac{2}{9} a^{2}(t)+\frac{a(t) c(t)}{3 e^{2}(t)}+\frac{c^{\prime}(t)}{3 e^{2}(t)}-\frac{c^{2}(t)}{9 e^{4}(t)}
$$

and

$$
h(t)=\frac{1}{3} a(t)+\frac{2 c(t)}{3 e^{2}(t)} .
$$

Thus, equation (4.3) is transformed into a system of type (E).

As a simple example, let us consider the case in which $a(t)=a, b(t)=b$ and $c(t)=c$ for $t \geq 0$, where $a>0$ and $a b>c>0$. Then, $A(t)=a t$ and

$$
e^{2}(t)=\exp (-2 a t / 3)\left\{d+\frac{c}{a}(\exp (2 a t / 3)-1)\right\}
$$

for $t \geq 0$. Since $d$ is arbitrary and positive, we may take $c / a>0$ as $d$. Hence, it follows that $e(t)= \pm \sqrt{c / a}$. We also have

$$
g(t)=b-\frac{c}{a}-\frac{2}{9} a^{2}+\frac{1}{3} a^{2}-\frac{1}{9} a^{2}=b-\frac{c}{a}>0
$$

and

$$
h(t)=\frac{1}{3} a+\frac{2}{3} a=a>0
$$

for $t \geq 0$. Since $f(t)=1$, we get

$$
\psi(t)=2 h(t)+\frac{g^{\prime}(t)}{g(t)}=2 a>0
$$

for $t \geq 0$. Hence, assumptions (i)-(iv) in Theorem 2.1 are satisfied.

If $a(t)$ and $b(t)$ are twice differentiable, and $b(t)$ is differentiable for $t \geq 0$, then $g(t)$ is also differentiable for $t \geq 0$, and hence, $\psi(t)$ is well defined. Thus, we have the following result.

Corollary 4.1. Let $f(t)=1$ for $t \geq 0$ and let $e(t), g(t)$ and $h(t)$ be given by (4.4), (4.5) and (4.6), respectively. Suppose that assumptions (i)-(iv) are satisfied. Then the equilibrium $\left(x, x^{\prime}, x^{\prime \prime}\right)=(0,0,0)$ of (4.3) is asymptotically stable. 
In our main theorem, Theorem 2.1, we assumed the boundedness of all coefficients $e(t), f(t), g(t)$ and $h(t)$ of $(E)$. If

$$
|e(t)|>0 \text { for } t \geq 0
$$

then we can relax the boundedness. In order to explain this, we define

$$
\tau=E(t)=\int_{0}^{t}|e(s)| d s
$$

and assume that

$$
E(t) \rightarrow \infty \quad \text { as } t \rightarrow \infty .
$$

From (4.7) and (4.8) it turns out that $\tau \rightarrow \infty$ if and only if $t \rightarrow \infty$. Also, we have

$$
\frac{d x}{d \tau}=\frac{e(t)}{|e(t)|} y, \quad \frac{d y}{d \tau}=-\frac{e(t)}{|e(t)|} x+\frac{f(t)}{|e(t)|} z, \quad \frac{d z}{d \tau}=-\frac{g(t)}{|e(t)|} y-\frac{h(t)}{|e(t)|} z,
$$

where $t=E^{-1}(\tau)$. Let $\tilde{e}(t)=e(t) /|e(t)|, \tilde{f}(t)=f(t) /|e(t)|, \tilde{g}(t)=g(t) /|e(t)|$ and $\tilde{h}(t)=h(t) /|e(t)|$ for $t \geq 0$. Then we see that system (4.9) has the form of (E). Note that $\tilde{e}(t)=1$ or $\tilde{e}(t)=-1$ for $t \geq 0$. Since $g(t) / f(t)$ is differentiable for $t \geq 0$, then we have

$$
\tilde{\psi}(t) \stackrel{\text { def }}{=} 2 \tilde{h}(t)+\frac{\tilde{f}(t)}{\tilde{g}(t)}\left(\frac{\tilde{g}(t)}{\tilde{f}(t)}\right)^{\prime}=\frac{2 h(t)}{|e(t)|}+\frac{f(t)}{g(t)}\left(\frac{g(t)}{f(t)}\right)^{\prime}
$$

for $t \geq 0$. Assumption (iii) is always satisfied, because $\tilde{e}(t)=1$ or $\tilde{e}(t)=-1$ for $t \geq 0$. Thus, we have the following results.

Theorem 4.2. Let (4.7) and (4.8) hold. Suppose that $\tilde{f}(t), \tilde{g}(t)$ and $\tilde{h}(t)$ are bounded for $t \geq 0$. If

(v) $\tilde{f}(t) \tilde{g}(t)>0$ for $t \geq 0$ and $\liminf _{t \rightarrow \infty} \tilde{f}(t) \tilde{g}(t)>0$,

(vi) $\int_{0}^{\infty} \tilde{\psi}_{-}(s) d s<\infty$,

(vii) $\tilde{\psi}_{+}(t)$ is weakly integrally positive, then the zero solution of $(E)$ is asymptotically stable.

Corollary 4.3. Let (4.7) and (4.8) hold and let $e(t), g(t)$ and $h(t)$ be given by (4.4), (4.5) and (4.6), respectively. Suppose that $1 /|e(t)|, g(t) /|e(t)|$ and $h(t) /|e(t)|$ are bounded for $t \geq 0$. If assumptions (v)-(vii) are satisfied, then the equilibrium $\left(x, x^{\prime}, x^{\prime \prime}\right)=(0,0,0)$ of (4.3) is asymptotically stable. 
Example 4.2. Consider system (E) with

$$
e(t)=f(t)=g(t)=1+t \quad \text { and } \quad h(t)=\sin ^{2} t .
$$

Then the zero solution of $(E)$ is asymptotically stable.

Since $e(t)>1$ for $t \geq 0$ and $E(t)$ tends to $\infty$ as $t \rightarrow \infty$, conditions (4.7) and (4.8) hold. In this example, $\tilde{f}(t)=\tilde{g}(t)=1$ and $\tilde{h}(t)=\sin ^{2} t /(1+t)$ for $t \geq 0$. Hence, these are bounded for $t \geq 0$ and assumption (v) is satisfied. Since

$$
\tilde{\psi}(t)=\frac{2 h(t)}{|e(t)|}+\frac{f(t)}{g(t)}\left(\frac{g(t)}{f(t)}\right)^{\prime}=\frac{2 \sin ^{2} t}{1+t}>0
$$

for $t \geq 0$, we see that $\tilde{\psi}_{+}(t)=2 \sin ^{2} t /(1+t)$ and $\tilde{\psi}_{-}(t)=0$ for $t \geq 0$, and therefore, assumptions (vi) and (vii) are also satisfied. Thus, by Theorem 4.2, we conclude that the zero solution of $(E)$ is asymptotically stable. However, since $e(t), f(t)$ and $g(t)$ are unbounded, Theorem 2.1 is of no use, although assumptions (i)-(iv) are satisfied.

Figure 2 below indicates the positive orbit starting from the point $(0.8,0.8,1)$ at the initial time $t_{0}=0$ and its projections onto the $(x, y)$-plane, the $(y, z)$-plane and the $(x, z)$ plane. The positive orbit rotates about the origin infinitely many times and approaches the origin.

We take another example to illustrate Theorem 4.2.

EXAmple 4.3. Consider system (E) with

$$
e(t)=f(t)=g(t)=\frac{1}{1+t} \quad \text { and } \quad h(t)=\frac{1}{(1+t)^{2}}
$$

Then the zero solution of $(E)$ is asymptotically stable.

Since $e(t)=1 /(1+t)$ and $E(t)=\log (1+t)$ for $t \geq 0$, conditions (4.7) and (4.8) hold. It is clear that $\tilde{f}(t)=\tilde{g}(t)=1, \tilde{h}(t)=1 /(1+t)$ and $\tilde{\psi}(t)=2 /(1+t)$ for $t \geq 0$. Hence, we can easily confirm that $\tilde{f}(t), \tilde{g}(t)$ and $\tilde{h}(t)$ are bounded for $t \geq 0$ and assumptions (v)-(vii) are satisfied. Thus, by means of Theorem 4.2, we see that the zero solution of (E) is asymptotically stable.

In Example 4.3, $f(t) g(t)=1 /(1+t)^{2}$ and $\psi_{+}(t)=2 /(1+t)^{2}$ for $t \geq 0$. Hence, $f(t) g(t)$ tends to zero as $t \rightarrow \infty$ and $\psi_{+}(t)$ is not weakly integrally positive. Also, $e(t)$ tends to zero as $t \rightarrow \infty$, and there exists no triplex $(\alpha, \beta, \gamma)$ with $0<\alpha \leq \beta$ and $\gamma>0$ such that $I_{t}(\alpha, \beta, \gamma)$ is nonempty for $t$ sufficiently large. Thus, assumptions (i), (iii) and (iv) are not satisfied, and therefore, Theorem 2.1 is inapplicable to this example (we can verify assumption (ii) and the boundednessof $e(t), f(t), g(t)$ and $h(t)$ ). 

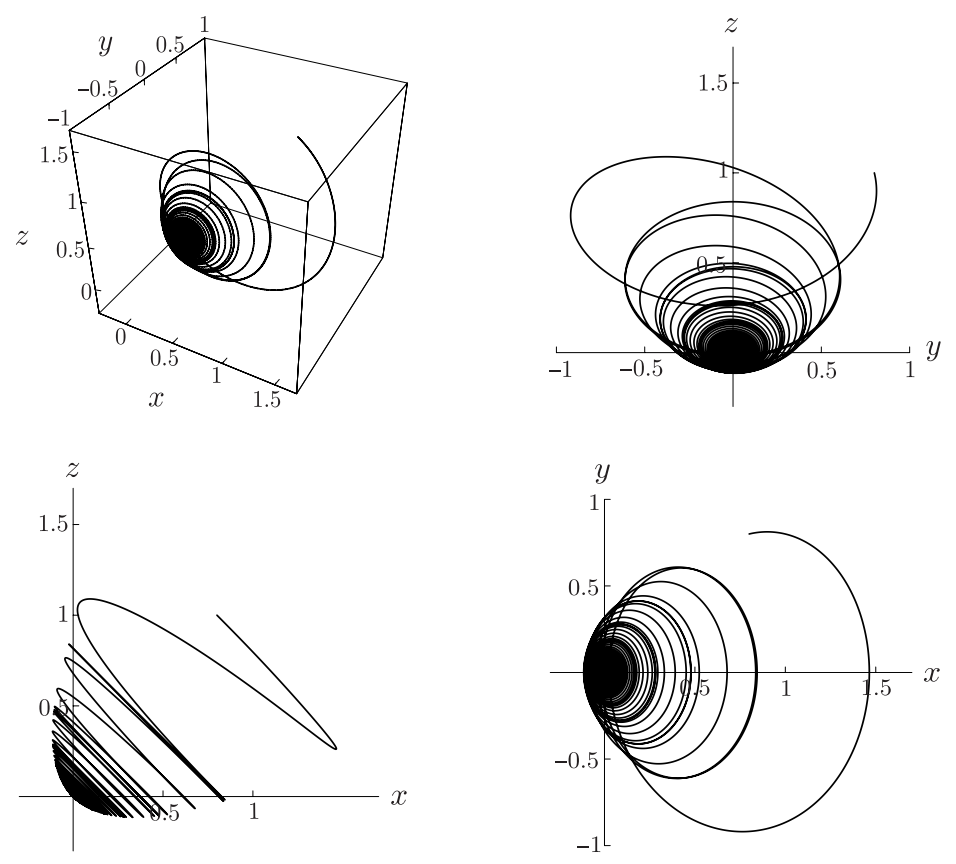

FIG. 2. A positive orbit of (E) with 4.10)

\section{REFERENCES}

[1] A. Halanay, Differential Equations: Stability, Oscillations, Time Lags, Academic Press, New York and London, 1966. MR0216103 (35:6938)

[2] J. K. Hale, Ordinary Differential Equations, Wiley-Interscience, New York, London and Sydney, 1969. MR0419901 (54:918) (revised) Krieger, Malabar, 1980. MR0587488 (82e:34001)

[3] L. Hatvani, A generalization of the Barbashin-Krasovskij theorems to the partial stability in nonautonomous systems, Qualitative Theory of Differential Equations I, II (ed. M. Farkas), Colloq. Math. Soc. János Bolyai 30, North-Holland, Amsterdam and New York, 1981, pp. 381-409. MR0680604 (84d:34048)

[4] L. Hatvani, On the uniform attractivity of solutions of ordinary differential equations by two Lyapunov functions, Proc. Japan Acad. 67, 162-167 (1991). MR.1114963 (92g:34063)

[5] L. Hatvani, On the asymptotic stability for a two-dimensional linear nonautonomous differential system, Nonlinear Anal. 25, 991-1002 (1995). MR1350721 (96k:34105)

[6] A. Hurwitz, Über die Bedingungen, unter welchen eine Gleichung nur Wurzeln mit negativen reellen Theilen besitzt, Math. Ann. 46, 273-284 (1895). MR1510884

[7] J. P. LaSalle and S. Lefschetz, Stability by Liapunov's Direct Method with Applications, Mathematics in Science and Engineering 4, Academic Press, New York and London, 1961. MR0132876 (24:A2712)

[8] J. C. Maxwell, On governors, Proc. Roy. Soc. London 6, 270-283 (1868).

[9] D. R. Merkin, Introduction to the Theory of Stability, Texts in Applied Mathematics 24, SpringerVerlag, New York, Berlin and Heidelberg, 1997. MR.1418401 (98f:34074)

[10] K. P. Persidskiı̌, Über die Stabilität einer Bewegung nach der ersten Näherung, Mat. Sb. 40, 284293 (1933).

[11] N. Rouche, P. Habets, and M. Laloy, Stability Theory by Liapunov's Direct Method, Applied Mathematical Sciences 22, Springer-Verlag, New York, Heidelberg and Berlin, 1977. MR0450715 (56:9008)

[12] E. J. Routh, Treatise on the Stability of a Given State of Motion, Macmillan, London, 1877. 
[13] J. Sugie, Influence of anti-diagonals on the asymptotic stability for linear differential systems, to appear in Monatsh. Math.

[14] T. Yoshizawa, Stability Theory by Liapunov's Second Method, Math. Soc. Japan, Tokyo, 1966. MR0208086 (34:7896) 